\title{
A gauge approach to an ordinal index of Baire one functions
}

\author{
by
}

\author{
Denny H. Leung (Singapore), Wee-Kee Tang (Singapore) and \\ Atok Zulijanto (Yogyakarta)
}

\begin{abstract}
We develop a calculus for the oscillation index of Baire one functions using gauges analogous to the modulus of continuity.
\end{abstract}

1. Introduction. Let $X$ be a metrizable space. A real-valued function $f$ is said to be of Baire class one (or simply, a Baire 1 function) if it is the pointwise limit of a sequence of continuous functions on $X$. The Baire Characterization Theorem states that if $X$ is Polish (separable completely metrizable), then $f: X \rightarrow \mathbb{R}$ is of Baire class one if and only if $f_{\mid F}$ has a point of continuity for every nonempty closed subset $F$ of $X$. Recently, Lee, Tang and Zhao [5] provided a characterization of Baire 1 functions in terms of gauges analogous to the modulus of continuity for continuous functions.

Theorem 1 ([5]). Suppose that $f: X \rightarrow \mathbb{R}$ is a real-valued function on a complete separable metric space $(X, d)$. Then the function $f$ is of Baire class one if and only if for any $\varepsilon>0$, there exists a positive function $\delta$ on $X$ such that

$$
|f(x)-f(y)|<\varepsilon \quad \text { whenever } \quad d(x, y)<\delta(x) \wedge \delta(y) .
$$

The Baire Characterization Theorem can be naturally quantified in terms of the oscillation index of Baire 1 functions [2]. This ordinal index was used in [3] to give a fine classification of Baire 1 functions. This line of investigation was continued by various authors: see e.g., [1], 4], [6], 7]. In this paper, we develop a method to compute the oscillation index of a Baire 1 function. The advantage of this approach is that it provides an easy-to-use calculus for the oscillation index that enables us to recover and refine all previously known results.

2010 Mathematics Subject Classification: Primary 26A21; Secondary 03E15, 54C30. Key words and phrases: Baire 1 functions, gauges, oscillation index. 
Let $\mathcal{C}$ denote the collection of all closed subsets of $X$. A derivation on $\mathcal{C}$ is a map $\mathcal{D}: \mathcal{C} \rightarrow \mathcal{C}$ such that (i) $\mathcal{D}(P) \subseteq P$ for all $P \in \mathcal{C}$ and (ii) $\mathcal{D}(P) \subseteq$ $\mathcal{D}(Q)$ if $P \subseteq Q$. A derivation $\mathcal{D}$ may be iterated in the usual manner. Let $\mathcal{D}^{0}(P)=P$. For all $\alpha<\omega_{1}$, let

$$
\mathcal{D}^{\alpha+1}(P)=\mathcal{D}\left(\mathcal{D}^{\alpha}(P)\right) \text {. }
$$

If $\alpha$ is a countable limit ordinal, set

$$
\mathcal{D}^{\alpha}(P)=\bigcap_{\gamma<\alpha} \mathcal{D}^{\gamma}(P) .
$$

The index of $\mathcal{D}$, denoted by $\iota(\mathcal{D})$, is the least countable ordinal $\alpha$ such that $\mathcal{D}^{\alpha}(X)=\emptyset$, if such an $\alpha$ exists, and $\omega_{1}$ otherwise.

Let $\varepsilon>0$ and a function $f: X \rightarrow \mathbb{R}$ be given. For any $P \in \mathcal{C}$, let $\mathcal{D}(f, \varepsilon, P)$ be the set of all $x \in P$ such that for any neighborhood $U$ of $x$, there exist $x_{1}, x_{2} \in P \cap U$ such that $\left|f\left(x_{1}\right)-f\left(x_{2}\right)\right| \geq \varepsilon$. For fixed $f$ and $\varepsilon>0, \mathcal{D}(f, \varepsilon, \cdot)$ is clearly a derivation on $\mathcal{C}$. Set $\beta(f, \varepsilon)=\iota(\mathcal{D}(f, \varepsilon, \cdot))$. The oscillation index of $f$ is $\beta(f)=\sup _{\varepsilon>0} \beta(f, \varepsilon)$. It is clear from the Baire Characterization Theorem that a real-valued function on a complete separable metric space is Baire 1 if and only if its oscillation index is countable. For a countable ordinal $\xi$, let $\mathfrak{B}_{1}^{\xi}(X)$ denote the set of all Baire 1 functions on $X$ with $\beta(f) \leq \omega^{\xi}$.

Let $\pi: X \rightarrow \mathbb{R}$ be a function that is never zero. For any closed subset $H$ of $X$ let $\mathcal{Z}(\pi, H)$ be the set of all $x \in H$ such that for any neighborhood $U$ of $x, \inf \{|\pi(y)|: y \in U \cap H\}=0$. Clearly, given a fixed $\pi, \mathcal{Z}(\pi, \cdot)$ is a derivation on $\mathcal{C}$. We define the zero index $o(\pi)$ of $\pi$ to be the index of the derivation $\mathcal{Z}(\pi, \cdot)$.

We conclude this section by stating two simple facts concerning derivations that will be used below. They are easily verified by using transfinite induction.

Proposition 2. Let $\mathcal{D}$ and $\mathcal{E}$ be derivations.

(1) If $\mathcal{D} P \subseteq \mathcal{E} P$ for all $P$, then $\mathcal{D}^{\alpha} P \subseteq \mathcal{E}^{\alpha} P$ for all $P$ and all $\alpha<\omega_{1}$. Hence $\iota(\mathcal{D}) \leq \iota(\mathcal{E})$.

(2) Suppose that $\mathcal{D}(P \cup Q) \subseteq \mathcal{D}(P) \cup \mathcal{D}(Q)$ for all $P$ and $Q$. Then $\mathcal{D}^{\alpha}(P \cup Q) \subseteq \mathcal{D}^{\alpha}(P) \cup \mathcal{D}^{\alpha}(Q)$ for all $P, Q$ and all $\alpha<\omega_{1}$.

2. Gauges and their zero indices. Let $f$ be a real-valued function on a separable complete metric space $(X, d)$ and let $\varepsilon>0$. A positive function $\delta: X \rightarrow \mathbb{R}^{+}$is called an $\varepsilon$-gauge of $f$ if for any $x, y \in X$,

$$
|f(x)-f(y)|<\varepsilon \quad \text { whenever } \quad d(x, y)<\delta(x) \wedge \delta(y) .
$$


One can easily check that if $\delta$ is an $\varepsilon$-gauge of $f$, then $\mathcal{D}(f, \varepsilon, P) \subseteq \mathcal{Z}(\delta, P)$ for every closed set $P$. Applying Proposition 2(1), we obtain the following immediately.

Proposition 3. Let $\varepsilon>0$ and a Baire 1 function $f: X \rightarrow \mathbb{R}$ be given. If $\delta: X \rightarrow \mathbb{R}^{+}$is an $\varepsilon$-gauge of $f$, then $\beta(f, \varepsilon) \leq o(\delta)$.

The utility of the gauge approach comes from the fact that the inequality in Proposition 3 can be made into an equality.

Theorem 4. Let $f: X \rightarrow \mathbb{R}$ be a Baire 1 function. Then for any $\varepsilon>0$ there exists an $\varepsilon$-gauge $\delta$ of $f$ such that $o(\delta)=\beta(f, \varepsilon)$.

Proof. For simplicity, write $\mathcal{D}^{\alpha}$ for $\mathcal{D}^{\alpha}(f, \varepsilon, X)$ for all $\alpha<\omega_{1}$. For all $\alpha<$ $\beta(f)$ and $x \in \mathcal{D}^{\alpha} \backslash \mathcal{D}^{\alpha+1}$, define $\delta(x)$ to be the supremum of all $\delta$ such that $\delta \leq \min \left\{1, d\left(x, \mathcal{D}^{\alpha+1}\right)\right\}$ and $\left|f\left(x_{1}\right)-f\left(x_{2}\right)\right|<\varepsilon$ for all $x_{1}, x_{2} \in B(x, \delta) \cap \mathcal{D}^{\alpha}$. By definition of $\mathcal{D}^{\alpha}$ and $\mathcal{D}^{\alpha+1}, \delta(x)>0$. We first show that $\delta$ is an $\varepsilon$-gauge of $f$. Indeed, for $x, y \in X$ with $d(x, y)<\delta(x) \wedge \delta(y)$, we may assume that $x \in \mathcal{D}^{\alpha} \backslash \mathcal{D}^{\alpha+1}, y \in \mathcal{D}^{\gamma} \backslash \mathcal{D}^{\gamma+1}$, with $\alpha \leq \gamma$. Then $d(x, y)<\delta(x) \leq d\left(x, \mathcal{D}^{\alpha+1}\right)$ and hence $y \notin \mathcal{D}^{\alpha+1}$. Thus $\alpha=\gamma$. Choose $\delta_{0}$ such that $d(x, y)<\delta_{0}<\delta(x)$. We have $x, y \in B\left(x, \delta_{0}\right) \cap \mathcal{D}^{\alpha}$ and hence $|f(x)-f(y)|<\varepsilon$. This proves that $\delta$ is an $\varepsilon$-gauge for $f$. By Proposition $3, \beta(f, \varepsilon) \leq o(\delta)$.

We prove the reverse inequality by showing inductively that $\mathcal{Z}^{\alpha}(\delta, X) \subseteq$ $\mathcal{D}^{\alpha}$ for all $\alpha \leq \beta(f)$. The claim is obvious if $\alpha$ is 0 or a limit ordinal. Suppose that $\mathcal{Z}^{\alpha}(\delta, X) \subseteq \mathcal{D}^{\alpha}$ for some $\alpha<\beta(f)$. Take any $x \in \mathcal{Z}^{\alpha}(\delta, X) \backslash \mathcal{D}^{\alpha+1}$. By the inductive hypothesis, $x \in \mathcal{D}^{\alpha} \backslash \mathcal{D}^{\alpha+1}$. Choose $\delta_{0}$ so that $0<\delta_{0}<\delta(x)$. For any $y \in B\left(x, \delta_{0}\right) \cap \mathcal{Z}^{\alpha}(\delta, X) \subseteq \mathcal{D}^{\alpha} \backslash \mathcal{D}^{\alpha+1}$, let $\delta^{\prime}=\delta_{0}-d(x, y)$. Then $0<\delta^{\prime} \leq \delta(x) \leq 1$. Also,

$$
d\left(y, \mathcal{D}^{\alpha+1}\right) \geq d\left(x, \mathcal{D}^{\alpha+1}\right)-d(x, y)>\delta_{0}-d(x, y)=\delta^{\prime} .
$$

If $x_{1}, x_{2} \in B\left(y, \delta^{\prime}\right) \cap \mathcal{D}^{\alpha}$, then $x_{1}, x_{2} \in B\left(x, \delta_{0}\right) \cap \mathcal{D}^{\alpha}$ and thus we have $\left|f\left(x_{1}\right)-f\left(x_{2}\right)\right|<\varepsilon$. By definition, $\delta(y) \geq \delta^{\prime}=\delta_{0}-d(x, y)$. This proves that $x \notin \mathcal{Z}^{\alpha+1}(\delta, X)$ and completes the induction.

3. Some computational tools. Before we see some applications of Theorem 4, we establish some computational tools for estimating the zero index. The next lemma is implicitly contained in the proof of [3, Lemma 5].

Lemma 5. Let $\mathcal{D}_{1}$ and $\mathcal{D}_{2}$ be derivations. If $\mathcal{D}$ is a derivation so that $\mathcal{D}(P \cup Q) \subseteq \mathcal{D}(P) \cup \mathcal{D}(Q)$ and $\mathcal{D}(P) \subseteq \mathcal{D}_{1}(P) \cup \mathcal{D}_{2}(P)$ for all $P$ and $Q$, then $\mathcal{D}^{\omega^{\xi}}(P) \subseteq \mathcal{D}_{1}^{\omega^{\xi}}(P) \cup \mathcal{D}_{2}^{\omega^{\xi}}(P)$ for $\xi<\omega_{1}$ and all $P$.

Proof. We prove the lemma by induction on $\xi$. By hypothesis, the statement holds for $\xi=0$. Since the sequences $\left(\mathcal{D}_{i}^{\alpha} P\right)_{\alpha}, i=1,2$, are nonincreasing, the inductive step for a limit ordinal $\xi$ is clear. Assume that the 
statement holds for some $\xi<\omega_{1}$. We need to show that

$$
\mathcal{D}^{\omega^{\xi+1}}(P) \subseteq \mathcal{D}_{1}^{\omega^{\xi+1}}(P) \cup \mathcal{D}_{2}^{\omega^{\xi+1}}(P) .
$$

To this end, we prove that

$$
\mathcal{D}^{\omega^{\xi} \cdot 2 n}(P) \subseteq \mathcal{D}_{1}^{\omega^{\xi} \cdot n}(P) \cup \mathcal{D}_{2}^{\omega^{\xi} \cdot n}(P)
$$

for all $n \in \mathbb{N}$. For each $s \in 2^{k}=\left\{\left(\varepsilon_{1}, \ldots, \varepsilon_{k}\right): \varepsilon_{i}=1\right.$ or 2$\}, k \in \mathbb{N}$, define $P_{s}$ as follows:

$$
P_{1}=\mathcal{D}_{1}^{\omega^{\xi}}(P), \quad P_{2}=\mathcal{D}_{2}^{\omega^{\xi}}(P)
$$

and

$$
P_{s^{\wedge} 1}=\mathcal{D}_{1}^{\omega^{\xi}}\left(P_{s}\right), \quad P_{s^{\wedge} 2}=\mathcal{D}_{2}^{\omega^{\xi}}\left(P_{s}\right) .
$$

To prove (3.1), we first prove that

$$
\mathcal{D}^{\omega^{\xi} \cdot k}(P) \subseteq \bigcup_{s \in 2^{k}} P_{s}
$$

for every $k \in \mathbb{N}$. By the inductive assumption, $(3.2)$ is true for $k=1$. Assume that it is true for some $k \in \mathbb{N}$. Then

$$
\begin{aligned}
\mathcal{D}^{\omega^{\xi} \cdot(k+1)}(P) & =\mathcal{D}^{\omega^{\xi}}\left(\mathcal{D}^{\omega^{\xi} \cdot k}(P)\right) \subseteq \mathcal{D}^{\omega^{\xi}}\left(\bigcup_{s \in 2^{k}} P_{s}\right) \\
& \subseteq \bigcup_{s \in 2^{k}} \mathcal{D}^{\omega^{\xi}}\left(P_{s}\right) \quad \text { by Proposition } 2(2) \\
& \subseteq \bigcup_{s \in 2^{k}}\left(\mathcal{D}_{1}^{\omega^{\xi}}\left(P_{s}\right) \cup \mathcal{D}_{2}^{\omega^{\xi}}\left(P_{s}\right)\right) \quad \text { by the inductive hypothesis } \\
& =\left(\bigcup_{s \in 2^{k}} P_{s^{\wedge} 1}\right) \cup\left(\bigcup_{s \in 2^{k}} P_{s^{\wedge} 2}\right)=\bigcup_{s \in 2^{k+1}} P_{s} .
\end{aligned}
$$

Thus 3.2 is verified by induction. Therefore, for all $n \in \mathbb{N}$,

$$
\begin{aligned}
\mathcal{D}^{\omega^{\xi} \cdot 2 n}(P) \subseteq & \bigcup_{s \in 2^{2 n}} P_{s} \\
\subseteq & \bigcup\left\{P_{s}: s \in 2^{2 n} \text { and } \operatorname{card}(\{k: s(k)=1\}) \geq n\right\} \\
& \cup \bigcup\left\{P_{s}: s \in 2^{2 n} \text { and } \operatorname{card}(\{k: s(k)=2\}) \geq n\right\} .
\end{aligned}
$$

If $s$ takes the value 1 at least $n$ times, then $P_{s} \subseteq \mathcal{D}_{1}^{\omega^{\xi} \cdot n}(P)$. Similarly if $s$ takes the value 2 at least $n$ times, then $P_{s} \subseteq \mathcal{D}_{2}^{\omega^{\xi} \cdot n}(P)$. Thus $(3.1)$ is proved.

Taking intersection over all $n$ in (3.1) gives

$$
\mathcal{D}^{\omega^{\xi+1}}(P) \subseteq \mathcal{D}_{1}^{\omega^{\xi+1}}(P) \cup \mathcal{D}_{2}^{\omega^{\xi+1}}(P)
$$


TheOREM 6. If $\pi_{1}, \pi_{2}: X \rightarrow \mathbb{R}$ are positive functions with $o\left(\pi_{1}\right) \leq \omega^{\xi}$ and $o\left(\pi_{2}\right) \leq \omega^{\xi}$ for some $\xi<\omega_{1}$, then $o\left(\pi_{1} \wedge \pi_{2}\right) \leq \omega^{\xi}$.

Proof. Let $\pi=\pi_{1} \wedge \pi_{2}$. Consider the derivations $\mathcal{D}(P)=\mathcal{Z}^{\alpha}(\pi, P)$, $\mathcal{D}_{i}(P)=\mathcal{Z}^{\alpha}\left(\pi_{i}, P\right), i=1,2$. It is easy to see that these derivations satisfy the hypotheses of Lemma 5. Therefore, the conclusion follows by the same lemma.

For any $\alpha<\omega_{1}$, set $[\alpha]=\inf \left\{\omega^{\xi}: \alpha \leq \omega^{\xi}\right\}$.

REMARK. For any Baire 1 function $f$, we may apply Theorems 4 and 6 to obtain $\delta_{f}: \mathbb{R} \times X \rightarrow \mathbb{R}^{+}$such that

(a) $\delta_{f}(\varepsilon, \cdot)$ is an $\varepsilon$-gauge for $f$,

(b) $\delta_{f}(\cdot, x)$ is a nondecreasing function for all $x \in X$,

(c) $o\left(\delta_{f}(\varepsilon, \cdot)\right) \leq[\beta(f)]$.

Indeed, according to Theorem 4 , for each $n \in \mathbb{N}$, there is an $1 / n$-gauge $\delta_{n}$ of $f$ such that $o\left(\delta_{n}\right)=\beta(f, 1 / n) \leq[\beta(f)]$. Set $\delta_{f}(1 / n, \cdot)=\delta_{1} \wedge \cdots \wedge \delta_{n}$. For all $x \in X,\left(\delta_{f}(1 / n, x)\right)_{n}$ is nonincreasing. Clearly, $\delta_{f}(1 / n, \cdot)$ is an $\varepsilon$-gauge for $f$ if $1 / n \leq \varepsilon$. Also by Theorem 6, $o\left(\delta_{f}(1 / n, \cdot)\right) \leq[\beta(f)]$. Given $\varepsilon>0$, set $\delta_{f}(\varepsilon, \cdot)=\delta_{f}(1 / n, \cdot)$ if $\varepsilon \in[1 / n, 1 /(n-1))(1 / 0=+\infty)$. Then $\delta_{f}$ has the desired properties. A function $\delta_{f}$ satisfying (a)-(c) will be called a $B$-gauge for $f$.

Proposition 7. Let $\phi$ and $\psi$ be positive functions.

(1) If there is $0<c<\infty$ such that $\psi \leq c \phi$, then $o(\phi) \leq o(\psi)$.

(2) $o\left(\phi^{2}\right)=o(\phi)$.

(3) $o(\phi \psi) \leq[o(\phi)] \vee[o(\psi)]$.

Proof. (1) and (2) are clear. For (3), observe that $(\phi \wedge \psi)^{2} \leq \phi \psi$. So by (1), (2) and Theorem 6 ,

$$
o(\phi \psi) \leq o\left((\phi \wedge \psi)^{2}\right)=o(\phi \wedge \psi) \leq[o(\phi)] \vee[o(\psi)] .
$$

Let $f$ be a real-valued function on $X$. For any closed subset $H$ of $X$, let

$$
\mathcal{U}(f, H)=\left\{x \in H: \limsup _{\substack{y \rightarrow x \\ y \in H}}|f(y)|=\infty\right\} .
$$

Then $\mathcal{U}(f, \cdot)$ is clearly a derivation on $\mathcal{C}$. The unboundedness index $u(f)$ of $f$ is defined as the index of the derivation $\mathcal{U}(f, \cdot)$.

The following proposition follows easily from the fact that $\mathcal{Z}(1 /(\phi+a), \cdot)$ $=\mathcal{U}(\phi, \cdot)$ for any $a>0$.

Proposition 8. Let $\phi$ be a positive function and $a>0$. Then

$$
o\left(\frac{1}{\phi+a}\right)=u(\phi) .
$$


4. Applications. It was shown in [3, Section 2] that $\mathcal{B}_{1}^{\xi}(K)$, the space of bounded Baire 1 functions $f$ on a compact metric space $K$ with $\beta(f) \leq \omega^{\xi}$, is a Banach algebra under pointwise operations. In [6], extension to unbounded Baire 1 functions was considered. It was found that for a compact metric space $K$, if $f \in \mathfrak{B}_{1}^{\xi_{1}}(K)$ and $g \in \mathfrak{B}_{1}^{\xi_{2}}(K)$ then $f+g \in \mathfrak{B}_{1}^{\xi_{1} \vee \xi_{2}}(K)$ and $f g \in \mathfrak{B}_{1}^{\xi}(K)$, where $\xi=\max \left\{\xi_{1}+\xi_{2}, \xi_{2}+\xi_{1}\right\}$. (See [6], Proposition 4.4 and Theorem 6.5 respectively.) In this section, we show that generalized versions of these results on a Polish space may be obtained via the gauge approach using Theorem 4 and the computational tools in $\S 3$. In fact, the gauge approach allows us to obtain estimates of the oscillation index of fairly general combinations of two Baire 1 functions.

Lemma 9. Suppose that $f: X \rightarrow \mathbb{R}$ is a Baire 1 function and $\delta_{f}$ is a $B$-gauge for $f$. Let $\xi=\sup _{\varepsilon>0} o\left(\delta_{f}(\varepsilon, \cdot)\right)$. If $\gamma: X \rightarrow \mathbb{R}$ is a positive function, then

$$
o\left(\delta_{f}(\gamma(\cdot), \cdot)\right) \leq \xi \cdot o(\gamma) .
$$

Proof. If $x \notin \mathcal{Z}(\gamma, H)$, then there exist $a>0$ and a neighborhood $U$ of $x$ such that $\gamma(y)>a$ for all $y \in U \cap H$. It follows from the monotonicity of $\delta_{f}$ in the first variable that

$$
U \cap \mathcal{Z}\left(\delta_{f}(\gamma(\cdot), \cdot), H\right) \subseteq \mathcal{Z}\left(\delta_{f}(a, \cdot), H\right) .
$$

By induction, we have

$$
U \cap \mathcal{Z}^{\alpha}\left(\delta_{f}(\gamma(\cdot), \cdot), H\right) \subseteq \mathcal{Z}^{\alpha}\left(\delta_{f}(a, \cdot), H\right)
$$

for all $\alpha<\omega_{1}$. In particular,

$$
U \cap \mathcal{Z}^{\xi}\left(\delta_{f}(\gamma(\cdot), \cdot), H\right)=\emptyset .
$$

It follows that $x \notin \mathcal{Z}^{\xi}\left(\delta_{f}(\gamma(\cdot), \cdot), H\right)$. Hence

$$
\mathcal{Z}^{\xi}\left(\delta_{f}(\gamma(\cdot), \cdot), H\right) \subseteq \mathcal{Z}(\gamma, H) .
$$

By Proposition 2(1), we have

$$
\mathcal{Z}^{\xi \cdot \alpha}\left(\delta_{f}(\gamma(\cdot), \cdot), H\right) \subseteq \mathcal{Z}^{\alpha}(\gamma, H)
$$

for all $\alpha<\omega_{1}$. In particular,

$$
\mathcal{Z}^{\xi \cdot o(\gamma)}\left(\delta_{f}(\gamma(\cdot), \cdot), X\right)=\emptyset .
$$

It follows that $o\left(\delta_{f}(\gamma(\cdot), \cdot)\right) \leq \xi \cdot o(\gamma)$.

Let $I, J \subseteq \mathbb{R}$, and let $f: X \rightarrow I$ and $g: X \rightarrow J$. Given $\varepsilon>0$, a function $F: I \times J \rightarrow \mathbb{R}$ is said to satisfy property $(*)_{\varepsilon}$ with respect to $(f, g)$ if there are $h_{1}: I \rightarrow \mathbb{R}$ and $h_{2}: J \rightarrow \mathbb{R}$ such that

$$
|F(f(x), g(x))-F(f(y), g(y))|<\varepsilon
$$


whenever

$$
|f(x)-f(y)|<h_{1}(x) \vee h_{1}(y) \quad \text { and } \quad|g(x)-g(y)|<h_{2}(x) \vee h_{2}(y) .
$$

TheOrem 10. Suppose that $f, g: X \rightarrow \mathbb{R}$ are functions of Baire class one with $f(X) \subseteq I$ and $g(X) \subseteq J$. If $F: I \times J \rightarrow \mathbb{R}$ satisfies property $(*)_{\varepsilon}$ with respect to $(f, g)$, then $\beta(F(f, g), \varepsilon) \leq[\beta(f)]\left[o\left(h_{1}\right)\right] \vee[\beta(g)]\left[o\left(h_{2}\right)\right]$.

Proof. Let $\varepsilon>0$ and let $h_{1}, h_{2}$ be given by property $(*)_{\varepsilon}$. Set $\delta(x)=$ $\delta_{f}\left(h_{1}(x), x\right) \wedge \delta_{g}\left(h_{2}(x), x\right)$, where $\delta_{f}$ and $\delta_{g}$ are B-gauges of $f$ and $g$ respectively. We first show that $\delta$ is an $\varepsilon$-gauge for $F(f, g)$. Indeed, if $d(x, y)<$ $\delta(x) \wedge \delta(y)$, then

$$
\begin{aligned}
d(x, y) & <\delta_{f}\left(h_{1}(x), x\right) \wedge \delta_{f}\left(h_{1}(y), y\right) \\
& \leq \delta_{f}\left(h_{1}(x) \vee h_{1}(y), x\right) \wedge \delta_{f}\left(h_{1}(x) \vee h_{1}(y), y\right) .
\end{aligned}
$$

Therefore,

$$
|f(x)-f(y)|<h_{1}(x) \vee h_{1}(y) .
$$

Likewise,

$$
|g(x)-g(y)|<h_{2}(x) \vee h_{2}(y)
$$

By property $(*)_{\varepsilon}$,

$$
|F(f(y), g(y))-F(f(x), g(x))|<\varepsilon .
$$

So $\delta$ is an $\varepsilon$-gauge for $F(f, g)$. It remains to estimate $o(\delta)$. By the definitions of $\delta_{f}$ and $\delta_{g}$,

$$
\sup _{\varepsilon>0} o\left(\delta_{f}(\varepsilon, \cdot)\right) \leq[\beta(f)] \quad \text { and } \quad \sup _{\varepsilon>0} o\left(\delta_{g}(\varepsilon, \cdot)\right) \leq[\beta(g)] .
$$

By Lemma 9 ,

$$
o\left(\delta_{f}\left(h_{1}(\cdot), \cdot\right)\right) \leq[\beta(f)]\left[o\left(h_{1}\right)\right] \quad \text { and } \quad o\left(\delta_{g}\left(h_{2}(\cdot), \cdot\right)\right) \leq[\beta(g)]\left[o\left(h_{2}\right)\right] .
$$

Therefore, by Theorem 6 .

$$
o(\delta)=o\left(\delta_{f}\left(h_{1}(\cdot), \cdot\right) \wedge \delta_{g}\left(h_{2}(\cdot), \cdot\right)\right) \leq[\beta(f)]\left[o\left(h_{1}\right)\right] \vee[\beta(g)]\left[o\left(h_{2}\right)\right] .
$$

The desired conclusion follows from Proposition 3 .

THEOREM 11. If $F: I \times J \rightarrow \mathbb{R}$ is uniformly continuous, then $\beta(F(f, g))$ $\leq[\beta(f)] \vee[\beta(g)]$.

Proof. Since $F$ is uniformly continuous on $I \times J$, for any $\varepsilon>0$, there are positive constant functions $h_{1}$ and $h_{2}$ that witness the fact that $F$ satisfies $(*)_{\varepsilon}$ with respect to $(f, g)$. By Theorem $10, \beta(F(f, g), \varepsilon) \leq[\beta(f)]\left[o\left(h_{1}\right)\right] \vee$ $[\beta(g)]\left[o\left(h_{2}\right)\right]=[\beta(f)] \vee[\beta(g)]$, as $o\left(h_{1}\right)=o\left(h_{2}\right)=1$. 
Corollary 12 ([3], Section 2], [6, Proposition 4.4]). If $f, g \in \mathfrak{B}_{1}^{\xi}(X)$, then $f+g,|f| \in \mathfrak{B}_{1}^{\xi}(X)$. Furthermore, when $f, g$ are bounded, $f g \in \mathfrak{B}_{1}^{\xi}(X)$.

Proof. Since $F_{1}(u, v)=u+v$ and $F_{2}(u, v)=|u|$ are uniformly continuous functions, the first assertion follows easily from Theorem 11. The second assertion is also clear as the product function is uniformly continuous on bounded sets.

The next result improves the estimate given in [6, Theorem 6.5].

Theorem 13. $\beta(f g) \leq[\beta(f)][u(g)] \vee[\beta(g)][u(f)]$.

Proof. Let $0<\varepsilon<1$. Then $F(u, v)=u v$ has property $(*)_{\varepsilon}$ with respect to $(f, g)$ with

$$
h_{1}(x)=\frac{\varepsilon}{3(|g(x)|+1)} \quad \text { and } \quad h_{2}(x)=\frac{\varepsilon}{3(|f(x)|+1)} .
$$

Indeed, if

$$
|f(x)-f(y)|<h_{1}(x) \vee h_{1}(y) \quad \text { and } \quad|g(x)-g(y)|<h_{2}(x) \vee h_{2}(y),
$$

then

$$
|f(x)-f(y)|<\frac{\varepsilon}{3(|g(x)|+1)} \vee \frac{\varepsilon}{3(|g(y)|+1)} \leq 1
$$

and

$$
|g(x)-g(y)|<\frac{\varepsilon}{3(|f(x)|+1)} \vee \frac{\varepsilon}{3(|f(y)|+1)} \leq 1 .
$$

Since $|g(y)| \leq|g(y)-g(x)|+|g(x)|<1+|g(x)|$, it follows that

$$
|g(y)||f(x)-f(y)| \leq \frac{\varepsilon|g(y)|}{3(|g(x)|+1)} \vee \frac{\varepsilon|g(y)|}{3(|g(y)|+1)}<\frac{\varepsilon}{2} .
$$

Similarly,

$$
|f(x)||g(x)-g(y)|<\frac{\varepsilon}{2} .
$$

Therefore,

$$
\begin{aligned}
|F(f(x), g(x))-F(f(y), g(y))| & =|f(x) g(x)-f(y) g(y)| \\
& \leq|g(y)||f(x)-f(y)|+|f(x)||g(x)-g(y)| \\
& <\varepsilon / 2+\varepsilon / 2=\varepsilon .
\end{aligned}
$$

Thus $F$ has property $(*)_{\varepsilon}$ with respect to $(f, g)$. By Theorem 10 and Proposition 8 ,

$$
\begin{aligned}
\beta(f g, \varepsilon) & \leq[\beta(f)]\left[o\left(\frac{\varepsilon}{3(|g|+1)}\right)\right] \vee[\beta(g)]\left[o\left(\frac{\varepsilon}{3(|f|+1)}\right)\right] \\
& =[\beta(f)][u(g)] \vee[\beta(g)][u(f)] .
\end{aligned}
$$

Taking supremum over all $\varepsilon>0$ completes the proof. 
Corollary 14 ([], Theorem 6.5]). If $\beta(f) \leq \omega^{\xi_{1}}$ and $\beta(g) \leq \omega^{\xi_{2}}$, then $\beta(f g) \leq \omega^{\xi}$, where $\xi=\left(\xi_{1}+\xi_{2}\right) \vee\left(\xi_{2}+\xi_{1}\right)$.

Proof. Since $u(f) \leq \beta(f)$ and $u(g) \leq \beta(g)$, the result follows easily.

Note that an example has been constructed in [6] to show that the above result is optimal.

Theorem 15. If $g(x) \neq 0$ for all $x \in X$, then

$$
\beta\left(\frac{f}{g}\right) \leq[\beta(f)][o(g)] \vee[\beta(g)]([o(g)] \vee[u(f)]) .
$$

Proof. Let $0<\varepsilon<1$. Then $F(u, v)=u / v$ has property $(*)_{\varepsilon}$ with respect to $(f, g)$ with

$$
h_{1}(x)=\frac{\varepsilon|g(x)|}{8} \wedge 1, \quad h_{2}(x)=\frac{\varepsilon}{3}\left(\frac{|g(x)|^{2}}{|f(x)|+4} \wedge|g(x)|\right) .
$$

Indeed, if

$$
|f(x)-f(y)|<h_{1}(x) \vee h_{1}(y) \quad \text { and } \quad|g(x)-g(y)|<h_{2}(x) \vee h_{2}(y),
$$

then $|f(x)|<|f(y)|+1$,

$$
|f(x)-f(y)|<\frac{\varepsilon|g(x)|}{8} \vee \frac{\varepsilon|g(y)|}{8} \text { and }|g(x)-g(y)|<\frac{|g(x)|}{3} \vee \frac{|g(y)|}{3} .
$$

It follows that

$$
\frac{1}{2}|g(y)| \leq|g(x)| \leq 2|g(y)|
$$

and thus

$$
\frac{|f(x)-f(y)|}{|g(y)|} \leq \frac{\varepsilon}{4} .
$$

Also, from $|g(x)-g(y)| \leq \frac{\varepsilon}{3} \frac{|g(x)|^{2}}{|f(x)|+4} \vee \frac{\varepsilon}{3} \frac{|g(y)|^{2}}{|f(y)|+4}$, it follows that

$$
\begin{aligned}
|f(x)||g(x)-g(y)| & \leq \frac{\varepsilon}{3} \frac{|g(x)|^{2}|f(x)|}{|f(x)|+4} \vee \frac{\varepsilon}{3} \frac{|g(y)|^{2}|f(x)|}{|f(y)|+4} \\
& \leq \frac{\varepsilon}{3}|g(x)|^{2} \vee \frac{\varepsilon}{3}|g(y)|^{2} \\
& \leq \frac{2 \varepsilon}{3}|g(x)||g(y)| \text { by 4.1. }
\end{aligned}
$$

Thus

$$
\frac{|f(x)||g(x)-g(y)|}{|g(x)||g(y)|} \leq \frac{2 \varepsilon}{3} .
$$


Therefore

$$
\begin{aligned}
\left|\frac{f(x)}{g(x)}-\frac{f(y)}{g(y)}\right| & \leq\left|\frac{f(x)}{g(x)}-\frac{f(x)}{g(y)}\right|+\left|\frac{f(x)}{g(y)}-\frac{f(y)}{g(y)}\right| \\
& =|f(x)|\left|\frac{1}{g(x)}-\frac{1}{g(y)}\right|+\frac{1}{|g(y)|}|f(x)-f(y)| \\
& =\frac{|f(x)||g(x)-g(y)|}{|g(x)||g(y)|}+\frac{|f(x)-f(y)|}{|g(y)|} \\
& \leq 2 \varepsilon / 3+\varepsilon / 4<\varepsilon .
\end{aligned}
$$

Hence $F(u, v)=u / v$ has property $(*)_{\varepsilon}$ with respect to $(f, g)$. By Theorems 10 and 6 and Propositions 7 and 8 .

$$
\begin{aligned}
\beta\left(\frac{f}{g}\right) & \leq[\beta(f)]\left[o\left(\frac{\varepsilon|g|}{8} \wedge 1\right)\right] \vee[\beta(g)]\left[o\left(\frac{\varepsilon}{3}\left(\frac{|g|^{2}}{|f|+4} \wedge|g|\right)\right)\right] \\
& \leq[\beta(f)][o(g)] \vee[\beta(g)]\left(\left[o\left(\frac{|g|^{2}}{|f|+4}\right)\right] \vee[o(g)]\right) \\
& \leq[\beta(f)][o(g)] \vee[\beta(g)](([o(g)] \vee[u(f)]) \vee[o(g)]) \\
& \leq[\beta(f)][o(g)] \vee[\beta(g)]([o(g)] \vee[u(f)]) .
\end{aligned}
$$

If $o\left(\pi_{1}\right)=m$ and $o\left(\pi_{2}\right)=n$ are both finite, then the proof of Theorem 6 yields $o\left(\pi_{1} \wedge \pi_{2}\right) \leq m+n-1$. Now suppose that $f$ and $g$ are Baire 1 functions with $\beta(f)=m$ and $\beta(g)=n$. By Theorem 4, for any $\varepsilon>0$, there are $\varepsilon / 2$-gauges $\delta_{f}$ and $\delta_{g}$ of $f$ and $g$ respectively such that $o\left(\delta_{f}\right) \leq m$ and $o\left(\delta_{g}\right) \leq n$. It is clear that $\delta=\delta_{f} \wedge \delta_{g}$ is an $\varepsilon$-gauge for $f+g$. Since $o(\delta)=o\left(\delta_{f} \wedge \delta_{g}\right) \leq m+n-1$, we see that $\beta(f+g, \varepsilon) \leq m+n-1$ for all $\varepsilon>0$. The same argument goes for $f \vee g, f \wedge g$, and for bounded $f$ and $g$, $f g$. This recovers Theorem 1.3 of [1].

Acknowledgements. The authors would like to thank the referee for suggestions to improve the exposition and for simplifications to the proof of Theorem 4 .

Research of the first author was partially supported by AcRF project no. R-146-000-130-112.

\section{References}

[1] F. Chaatit, V. Mascioni and H. P. Rosenthal, On functions of finite Baire index, J. Funct. Anal. 142 (1996), 277-295.

[2] R. Haydon, E. Odell and H. P. Rosenthal, On certain classes of Baire-1 functions with applications to Banach space theory, in: Functional Analysis (Austin, TX, 1987-89), Lecture Notes in Math. 1470, Springer, New York, 1991, 1-35.

[3] A. S. Kechris and A. Louveau, A classification of Baire class 1 functions, Trans. Amer. Math. Soc. 318 (1990), 209-236. 
[4] P. Kiriakouli, A classification of Baire-1 functions, ibid. 351 (1999), 4599-4609.

[5] P.-Y. Lee, W.-K. Tang and D. Zhao, An equivalent definition of functions of the first Baire class, Proc. Amer. Math. Soc. 129 (2000), 2273-2275.

[6] D. H. Leung and W.-K. Tang, Functions of Baire class one, Fund. Math. 179 (2003), $225-247$.

[7] —, - Extension of functions with small oscillation, ibid. 192 (2006), 183-193.

Denny H. Leung

Department of Mathematics

National University of Singapore

2 Science Drive 2

Singapore 117543

E-mail: matlhh@nus.edu.sg

Atok Zulijanto

Department of Mathematics

Faculty of Mathematics and Natural Sciences

Gadjah Mada University

Yogyakarta 55281, Indonesia

E-mail: atokzulijanto@ugm.ac.id
Wee-Kee Tang

Add-venture Learning

431 Ang Mo Kio Avenue 2

Singapore 567871

E-mail: weekee@add-venture.com.sg

Received 26 June 2008;

in revised form 4 November 2009 